\title{
THE GARDEN-OF-EDEN THEOREM FOR FINITE CONFIGURATIONS
}

\author{
S. AMOROSO AND G. COOPER
}

\begin{abstract}
In [1] Moore showed that the existence of mutually erasable configurations in a two-dimensional tessellation space is sufficient for the existence of Garden-of-Eden configurations. In [2] Myhill showed that the existence of mutually indistinguishable configurations is both necessary and sufficient for the existence of Garden-of-Eden configurations.

After redefining the basic concepts with some minor changes in terminology, and after restating the main results from [1] and [2], we shall establish the equivalence between the existence of mutually erasable configurations and the existence of mutually indistinguishable configurations. This implies that the converse of Moore's result is true as well. We then show that by limiting the universe to the set of all finite configurations of the tessellation array, both of the above conditions remain sufficient, but neither is then necessary. Finally, we establish a necessary and sufficient condition for the existence of Garden-of-Eden configurations when only finite configurations are considered.
\end{abstract}

\section{The tessellation structure and the Garden-of-Eden theorems.} The tessellation array, which was first used by Von Neumann [3] in obtaining his results on machine self-reproduction, can be visualized as an infinite two-dimensional Euclidean space divided into square cells, in the fashion of a checkerboard, where each cell can hold any symbol from a finite set $A$. We use the set $Z^{2}$ of ordered pairs of integers to name the cells in the tessellation array. An array configunation, i.e., a symbol placed in each cell, is formally a mapping $c: Z^{2} \rightarrow A$. The restriction of an array configuration $c$ to a subset $S$ of $Z^{2}$ will be denoted by $(c)_{S}$. We speak of this as the configuration of $S$ in array configuration $c$. Each cell will behave like a deterministic and synchronous finite-state machine, and the symbol in cell $(i, j)$ at time $t$ will depend on the symbol in cell $(i, j)$ at time $t-1$ as well as the symbols in certain neighboring cells at time $t-1$. In this paper, as in [1] and [2], we fix the neighbors of any cell to be those cells (including the cell itself) which have each of their coordinates differing by at most 1 from the coordinate of the given cell. Figure 1 shows the neighbors of cell $(i, j)$.

Received by the editors September 2, 1969 and, in revised form, November 3, 1969. AMS subject classifications. Primary 0288, 0280, 9440.

Key words and phrases. Garden-of-Eden, self-reproducing, automata, tessellation automata, cellular automata, iterative arrays. 


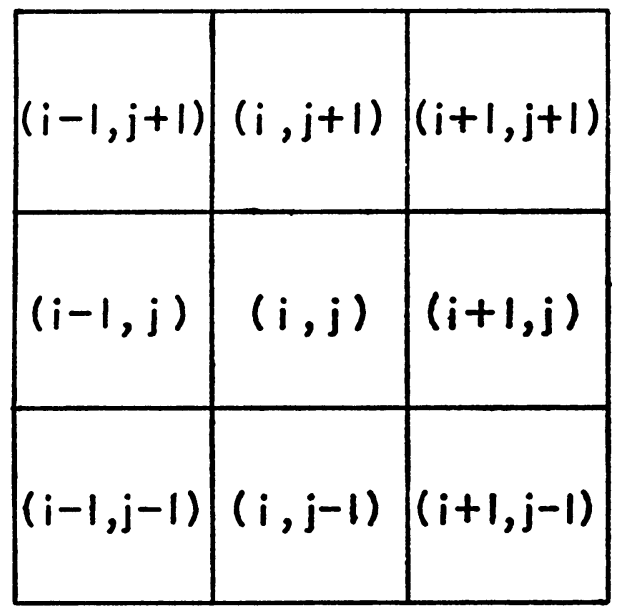

Figure 1

The function $\sigma$ which specifies the symbol to be placed in a cell at time $t$ given the symbols in the neighbors of the cell at time $t-1$ is to be the same for all cells in the tessellation array. This function $\sigma$, which we call a local transformation, acting on all cells simultaneously determines a global transformation $\tau$ which maps the set of all array configurations to the set of all array configurations. Any global transformation defined in this way, i.e., from a local transformation, will be referred to as a parallel transformation.

Moore's result. Let $S$ be an arbitrary finite nonempty subset of the set of cells of the tessellation array (i.e., $Z^{2}$ ). Let $N(S)$ be the set containing exactly all neighbors of any cell in $S$. Note $S \subset N(S)$.

For finite $S_{1} \subset Z^{2}$, a configuration $\left(c_{1}\right)_{S}$ where $S=N\left(N\left(S_{1}\right)\right)$ is said to be erasable if there exists an array configuration $c_{2}$ such that (a), (b), and (c) below hold.

(a) $\left(c_{1}\right)_{N\left(N\left(S_{1}\right)\right)-S_{1}}=\left(c_{2}\right)_{N\left(N\left(S_{1}\right)\right)-S_{1}}$,

(b) $\left(c_{1}\right)_{S_{1}} \neq\left(c_{2}\right)_{S_{1}}$, and

(c) $\left(\tau\left(c_{2}\right)\right)_{N\left(S_{1}\right)}=\left(\tau\left(c_{1}\right)\right)_{N\left(S_{1}\right)}$ 。

Such $c_{1}$ and $c_{2}$ will be said to be mutually erasable.

An array configuration $c$ is said to be a Garden-of-Eden (GOE) configuration if no array configuration $c^{\prime}$ exists such that $\tau\left(c^{\prime}\right)=c$.

Theorem 1. (MoORE's Garden-of-Eden Theorem). With respect to a given tessellation structure, if erasable configurations exist, then there exist Garden-of-Eden configurations.

The proof of this result is found in [1].

Myhill's result. Let $S$ be any finite nonempty set of cells. Distinct 
configurations $\left(c_{1}\right)_{S}$ and $\left(c_{2}\right)_{S}$ of $S$ are said to be indistinguishable if and only if the following holds: If for any finite set $E$ of cells that includes the set $S,\left(c_{1}\right)_{S}$ and $\left(c_{2}\right)_{S}$ are extended to array configurations $c_{1}$ and $c_{2}$ subject only to the requirement that $\left(c_{1}\right)_{E-S}=\left(c_{2}\right)_{E-S}$, then $\left(\tau\left(c_{1}\right)\right)_{N(S)}=\left(\tau\left(c_{2}\right)\right)_{N(S)}$.

THEOREM 2 (MyHILL). The existence of indistinguishable configurations is necessary and sufficient for the existence of Garden-of-Eden configurations.

The proof of this result is found in [2].1

The equivalence of the Moore and Myhill concepts of erasability. In [2], Myhill noted that by "weakening" the erasability requirement of Moore, he would be able to establish a necessary as well as sufficient condition for the existence of GOE configurations. ${ }^{2}$ However, since $E$ may be taken to be $N(N(S))$, indistinguishability trivially implies erasability; and since it follows at once from Theorems 1 and 2 that erasability implies indistinguishability, we have:

THEOREM 3. With respect to an arbitrary tessellation structure, the existence of erasable configurations is necessary and sufficient for the existence of indistinguishable configurations.

COROLlaRY 3.1. The existence of erasable configurations is necessary and sufficient for the existence of Garden-of-Eden configurations.

It might be noted that Myhill's argument for the variant of the converse of Moore's theorem can be applied almost without change to prove the converse of Moore's theorem directly.

II. The restriction to finite configurations. If we designate one symbol in $A$ as the quiescent symbol (usually, this will be denoted by 0 ), then we can define a finite configuration as an array configuration with only finitely many cells containing nonquiescent symbols. More precisely, $c: Z^{2} \rightarrow A$ is finite if the cardinality of $\left\{i \in Z^{2} \mid c(i) \in A-\{0\}\right\}$ is finite.

If parallel transformation $\tau$ is defined from a local transformation $\sigma$ which places the quiescent symbol in any cell at time $t$ if all its neighboring cells contain quiescent symbols at time $t-1$, then if $c$ is a finite configuration, so is $\tau(c)$. This condition on $\sigma$ is necessary and sufficient for preserving finite configurations.

1 Myhill established this result in [2] using the concept of "finite configuration," which we define later. However, this is unnecessary as can be seen in a later version of his paper [4] where this concept is not even introduced. He also redefines indistinguishable configurations in a slightly different way than in [2].

${ }^{2}$ In the later version, [4], Myhill no longer speaks of this alteration as a weakening. 
Moore stated in [1] that he wished to direct his attention exclusively to finite configurations, and indeed his proof of Theorem 1 is independent of whether the universe is the set of all configurations, or only the set of all finite configurations.

We now proceed to establish that the converse of Theorem 1 (whether stated in terms of erasable or indistinguishable configurations) is not true when the universe is limited to the set of all finite configurations. Hence, Myhill's converse requires the universe of all array configurations.

THEOREM 4. For an arbitrary tessellation structure, and with respect to the set of all finite configurations only, the existence of Garden-of-Eden configurations does not imply the existence of erasable or indistinguishable configurations.

Proof. The particular tessellation structure used in this proof will be one-dimensional. This is no essential limitation since it is a trivial matter to embed this in two dimensions. Alternatively, we might have defined the local transformation for the two-dimensional structure directly and have it actually depend only on certain neighbors. For a one-dimensional tessellation array we use the integers to name the cells. Consider the local transformation $\sigma_{1}$ defined by:

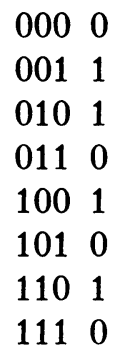

I.e., for any $i$, if at time $t$ cells $i-1, i$, and $i+1$ contain the respective symbols indicated in any line of the left column above, then at time $t+1$ cell $i$ will contain the corresponding symbol of the right column. We now proceed to show that $\sigma_{1}$ defines a parallel transformation $\tau_{1}$ on the set $C_{F}$ of all finite configurations that is one-to-one and properly into $C_{F}$. Hence, any finite configuration without a preimage under $\tau_{1}$ is a GOE configuration, and that no erasable or indistinguishable configurations exist follows easily from the fact that $\tau_{1}$ is one-to-one.

Consider an arbitrary finite configuration $c$ and suppose $c(i)=1$ and for all $j<i, c(j)=0$. If $\tau_{1}\left(c^{\prime}\right)=c$, then the definition of $\sigma_{1}$, the respective symbols in $c^{\prime}(i-1), c^{\prime}(i)$, and $c^{\prime}(i+1)$ must be one of the following: 
(a) 001,

(b) 010 ,

(c) 100 ,

(d) 110 .

That there is no such $c^{\prime}$ subject to case (c) follows from the fact that $\sigma_{1}(110)=\sigma_{1}(010)=1$ and $c(i-1)=0$. That no such $c^{\prime}$ exists subject to case (b) is established in a similar way. For case (d), with a little work, the reader can verify that $c(j)=0$ for all $j<i$ implies $c^{\prime}(k)=1$ for all $k<i+1$, hence no finite $c^{\prime}$ exists for this case.

We have established so far that only case (a) can hold and it is not difficult to see that if a finite $c^{\prime}$ exists such that $\tau_{1}\left(c^{\prime}\right)=c$ where $c(i)=1$ and $c(j)=0$ for all $j<i$, then $c^{\prime}(i+1)=1$ and $c^{\prime}(k)=0$ for all $k<i+1$. Hence, from the specification of $\sigma_{1}$ it is also easy to see that $c(i+1)$ uniquely determines $c^{\prime}(i+2), c(i+2)$ uniquely determines $c^{\prime}(i+3)$, etc. It follows from these remarks that for any finite configuration $c$, there can be at most one finite configuration $c^{\prime}$ such that $\tau_{1}\left(c^{\prime}\right)=c$.

To show that GOE configurations exist, consider the finite configuration $c$ defined as follows: $c(0)=1$ and $c(i)=0$ for all $i \neq 0$. From the remarks above we have that if a finite configuration $c^{\prime}$ exists such that $\tau_{1}\left(c^{\prime}\right)=c$ then $c^{\prime}(j)=0$ for all $j<1$, and $c^{\prime}(1)=1$. From the specification of $\tau_{1}, c(1)=0$ implies $c^{\prime}(2)=1, c(2)=0$ implies $c^{\prime}(3)=1$, etc. Therefore $c^{\prime}$ is not finite and hence $c$ is a GOE configuration, ${ }^{3}$ Q.E.D.

It is easily verified that under the restriction to $C_{F}$ any surjective (i.e., onto) parallel transformation is also bijective (i.e., one-to-one and onto). This is seen as follows. Suppose there could exist distinct $c_{1}, c_{2} \in C_{F}$ such that $\tau\left(c_{1}\right)=\tau\left(c_{2}\right)$ and $\tau$ is surjective. One could then easily define a finite set $S$ of cells (that would include all nonquiescent cells of $c_{1}$ and $c_{2}$ ) such that $\left(c_{1}\right)_{S}$ and $\left(c_{2}\right)_{S}$ would be mutually erasable, hence GOE configurations would exist and $\tau$ could not be surjective. This establishes:

TheOREM 5. With respect to $C_{F}$ and any arbitrary tessellation structure, $\tau$ is surjective iff it is bijective.

TheOREM 6. With respect to $C_{F}$ and any arbitrary tessellation structure, $\tau$ is not bijective iff Garden-of-Eden configurations exist.

Note that Theorems 5 and 6 are not true if the restriction to finite configurations is dropped. This can be seen by noting that $c_{1}$ defined

3 It can be shown for any tessellation structure that the existence of one GOE configuration implies the existence of infinitely many GOE configurations. 
by: $c_{1}(i)=0$ for all $i$, and $c_{2}$ defined by: $c_{2}(i)=1$ for all $i$, are both mapped by $\tau_{1}$ (from the proof of Theorem 4) to $c_{1}$, hence $\tau_{1}$ is not bijective on the set of all configurations. That for $\tau_{1}$ GOE configurations do not exist when the restriction to finite configurations is dropped can be seen as follows.

We shall say that a one-dimensional parallel transformation $\tau$ on a tessellation space of binary cells has property $R$ (property $L$ ) iff for any $a, b \in\{0,1\}, \sigma(0 a b) \neq \sigma(1 a b)(\sigma(a b 0) \neq \sigma(a b 1))$, where $\sigma$ defines $\tau$. With a little thought the reader should be able to see that any $\tau$ with property $R$ or $L$ can have no GOE configurations. $\tau_{1}$ has property $L$. (Additional details appear in [5].)

III. On Moore's concept of erasability. In his definition of erasable configurations of a set $S=N\left(N\left(S^{\prime}\right)\right)$ of cells, Moore used the cells in $N\left(N\left(S^{\prime}\right)\right)-S^{\prime}$ as two layers of "insulation" around the cells in $S^{\prime}$. We wish to establish here that Theorem 1 would not be true if Moore's definition were weakened by replacing $N\left(N\left(S^{\prime}\right)\right)$ by just $N\left(S^{\prime}\right)$, i.e. by using one layer of insulation rather than two.

Consider the parallel transformation $\tau_{2}$ on a one-dimensional array (again this can be trivially embedded in a two-dimensional structure) with binary cells defined by the local transformation $\sigma_{2}$ specified by:

$\begin{array}{ll}000 & 0 \\ 001 & 0 \\ 010 & 1 \\ 011 & 0 \\ 100 & 1 \\ 101 & 1 \\ 110 & 0 \\ 111 & 1\end{array}$

That $\tau_{2}$ is surjective with respect to $C_{F}$ can be established by showing that, for any $c \in C_{F}$, there is a $c^{\prime} \in C_{F}$ such that $\tau_{2}\left(c^{\prime}\right)=c$. Suppose $c(i)=1$ and, for all $k>i, c(k)=0$. Then let $c^{\prime}(i-1)=1$ and $c^{\prime}(k)=0$ for all $k>i-1$. From the definition of $\tau_{2}$ and since $\tau_{2}$ has property $R$, there will exist a $c^{\prime}$ such that $\tau_{2}\left(c^{\prime}\right)=c$. That $c^{\prime}$ is finite is seen as follows. If $c(j)=1$ and $c(k)=0$ for all $k<j$, then $c^{\prime}(j-1)$, $c^{\prime}(j)$ and $c^{\prime}(j+1)$ must contain respectively one of $010,100,101$, or 111 . In each case the reader can verify that $c(k)=0$ for all $k<j$ will require that $c^{\prime}$ be finite. This establishes that no GOE configurations can exist. Yet erasable configurations do exist (with respect to the weakened definition), e.g., let $S^{\prime}$ be a unit set, then the configurations 001 and 011 for $N\left(S^{\prime}\right)$ would be erasable. More precisely, if $\tau_{1}\left(c^{\prime}\right)=c$, then $c^{\prime}(i-2), c^{\prime}(i-1), c^{\prime}(i), c^{\prime}(i+1)$, and $c^{\prime}(i+2)$ containing respec- 
tively, 00010 or 00111 , would each result in $c(i-1), c(i)$, and $c(i+1)$ containing 001 .

IV. Concluding remarks. The reader trying to find the point where Myhill's proof [2] of the converse breaks down under the limitation to finite configurations, may find himself faced with an antinomy. The proof may seem independent of which universe of configurations, i.e. $C$ or $C_{F}$, is considered. The solution can be found in the following remark. For a given parallel transformation $\tau$, and with universe $C_{F}$, we called a configuration $c$ GOE if no finite $c^{\prime}$ existed such that $\tau\left(c^{\prime}\right)=c$. For Moore and Myhill, however, such a $c$ might not be GOE. For them $c$ is GOE only if no such $c^{\prime}$ exists, whether finite or not.

We might note further in closing, that each theorem stated above can be easily generalized to tessellation arrays of arbitrary dimension, and with a little more work, to neighborhoods of more general structure.

Acknowledgments. We wish to thank Professor Arthur W. Burks and an anonymous referee for the many improvements in our paper that resulted from their suggestions.

\section{REFERENCES}

1. Edward F. Moore, Machine models of self-reproduction, Proc. Sympos. Appl. Math., vol. 14, Amer. Math. Soc., Providence, R.I., 1962, pp. 17-33.

2. John Myhill, The converse to Moore's Garden-of-Eden theorem, Proc. Amer. Math. Soc. 14 (1963), 685-686. MR 27 \#5698.

3. John von Neumann, Theory of self-reproducing automata, Edited and completed by Arthur W. Burks, Univ. of Illinois Press, Urbana, 1966.

4. John Myhill, "The converse of Moore's Garden-of-Eden theorem," Essay 7 of Essays on cellular automata, Edited by Arthur W. Burks, Univ. of Illinois Press, Urbana, 1970.

5. Hisao Yamada and Serafino Amoroso, A completeness problem for pattern generation in tessellation automata, J. Comput. System Sci. 4 (1970), 137-176.

U.S. Army Electronics Command, Fort Monmouth, New Jersey 07703

Stevens Institute of Technology, Hoboken, New Jersey 07030

Princeton University, Princeton, New Jersey 08540 